\title{
The prevalence of temporomandibular joint involvement in rheumatoid arthri- tis patients: A cross-sectional study
}

\author{
Fahimeh Akhlaghi ${ }^{1}$, Shahabaldin Azizi ${ }^{2 *}$, Neda Amirimehr ${ }^{3}$ \\ ${ }^{1}$ Oral and Maxillofacial Surgery Department, School of dentistry, Taleghani Hospital, Shahid Beheshti University of \\ Medical Sciences, Tehran, Iran. ${ }^{2}$ Oral and Maxillofacial Surgery Dental School, Shahid Beheshti University of Medi- \\ cal Sciences, Tehran, Iran. ${ }^{3}$ Shahid Beheshti Universy of Medical Sciences, Tehran, Iran.
}

\begin{abstract}
Temporomandibular joint (TMJ) involvement in rheumatoid arthritis (RA) patients has been described in the literature; however, its incidence varies greatly. The current study aimed to determine the prevalence and clinical characteristics of TMJ involvement in RA patients.

This cross-sectional study consisted of 60 individuals with RA. The patients were assigned to two groups, those with TMJ involvement and those without TMJ involvement. TMJ involvement was defined based on the findings of questionnaires and clinical examinations by a rheumatologist. The symptoms and signs were recorded by a single trained dentist.

The mean age of patients was $43.9 \pm 15.52$ years (range: $21-78$ years), and the majority of the participants were women $(\mathrm{n}=49,81.66 \%)$. Thirty-five patients $(58.3 \%)$ had clinical evidence of TMJ involvement. The levels of C-reactive protein (CRP) $(P$ value $=0.01)$ and rheumatoid factor $(\mathrm{RF})(P$ value $=0.02)$ were found to be correlated with TMJ involvement () . There was no significant correlation between TMJ involvement and erythrocyte sedimentation rate (ESR), age, gender, treatment duration, or disease duration.

This study showed a high level of CRP in RA patients with TMJ involvement. The results further showed a significant correlation between TMJ involvement and RF. TMJ pain was reported in almost half of the RA patients.
\end{abstract}

Keywords: Temporomandibular joint, Rheumatoid arthritis, C-reactive protein, Rheumatoid factor

\section{Introduction}

Rheumatoid arthritis (RA) is a systemic disease characterized by chronic inflammation, joint swelling, joint tenderness, and destruction of synovial joints [1]. Overall, the prevalence of RA is $0.5-1 \%$ in the general population, and it is more frequent in women. RA is recognized as the most common inflammatory joint disorder [2]. It usually affects multiple joints of the body, often starting in the peripheral joints $[1,2]$. The temporomandibular joint (TMJ) is rarely the first joint to be affected [3]. The frequency of clinical TMJ involvement ranges from $5 \%$ to $86 \%$, with bilateral involvement reported as the most frequent $[4,5]$.

TMJ involvement in RA patients is excluded based on medical history, physical examination, radiographic findings, and laboratory test results. Therefore, a multidisciplinary approach is necessary [6]. Common clinical signs and symptoms of TMJ involvement are bilateral pain, swelling, stiffness during mouth opening, weakness of the masticatory muscles with decreased bite force, joint noises, and restriction of jaw movements [7]; in the late phase of
RA, ankylosis is more likely to occur [8]. The aim of this study was to evaluate the prevalence of TMJ involvement in RA patients.

\section{Materials and Methods}

This cross-sectional was approved by the Research Ethics Committee of Shahid Beheshti University of Medical Sciences under code number IR.SBMU.RIDs.REC. 1395.273. Sixty patients with RA were included in this study; the diagnosis of RA was based on criteria established by the American College of Rheumatology, 1987 [9]. The sample size was determined according to the most relevant study [10], and the sampling method was convenient sampling. The participants were selected consecutively among RA patients who referred to the outpatient clinic of Loghman-Hakim Hospital for diagnostic examination and treatment from January 2018 to July 2018. Those patients diagnosed with RA were informed about the study, and those who agreed to participate were included. The exclusion criteria were pregnancy; history of treatment for temporomandibular disorders (TMD); history of craniofa-

Personal non-commercial use only.Rheumatology Research Journal. Copyright (C) 2019. All rights reserved

*Corresponding Author: Shahabaldin Azizi, Oral and Maxillofacial Surgery Dental School, Shahid Beheshti University of Medical Sciences, Tehran, Iran, Email: shahabazizi44@gmail.com Tel: (+98)9120350641 Fax: (+98)021-88463760

Received: 21 May 2020; Accepted: 01 August 2020 
cial trauma, tumor, or infection of the orofacial and skull regions; and neoplasia in other locations. The general data of the participants, including gender, age, duration of disease, and treatment duration, were obtained from the patients' medical records. The blood samples taken from participants in both groups of this study were used to measure complete blood count (CBC), C-reactive protein (CRP), erythrocyte sedimentation rate (ESR), and rheumatoid factor (RF). ESR, CRP, and RF were considered as indicators of inflammation.

\section{Evaluation of TMJ involvement}

In the present study, TMJ involvement was defined based on the findings of questionnaires and clinical examinations by a rheumatologist. The symptoms and signs were recorded by a single trained dentist. The questionnaire used in this study was researcher-made and evaluated differential diagnoses in patients with RA. It included questions about the history of trauma, toothache, and dislocation. In the next step, the patients were classified into two groups:

Table 1. Clinical and laboratory findings of RA patients those with TMJ involvement $(\mathrm{N}: 35)$ and those without TMJ involvement (N: 25).

\section{Statistical Analysis}

The significance of differences between the two groups was analyzed by a t-test for normally distributed variables and by a Pearson chi-square for categorical variables; collected data was summarized as means $( \pm$ SD). Statistical analysis was performed using SPSS version 23; a $P$ value of $<0.05$ was considered statistically significant.

\section{Results}

A total of 60 RA patients were analyzed in this study. The majority of participants were female $(81.66 \%, n=49)$, and the mean age was $43.9 \pm 15.52$ years (range: $21-78$ years). The clinical and laboratory characteristics of RA patients are summarized in Table 1.

Of 60 RA patients examined, 35 (58.3\%) had clinical evidence of TMJ involvement (Table 2).

\begin{tabular}{cc}
\hline Male/Female (N\%) & 11 (18.34\%)/49 (81.66\%) \\
Age (years) & $43.9 \pm 15.52$ \\
RF (N\%) & Positive $48(80 \%)$ \\
CRP (mg/L) & Negative $12(20 \%)$ \\
ESR (mm/h) & $21.4 \pm 10.1$ \\
Disease duration (months) & $31.3 \pm 22.9$ \\
Treatment duration (months) & $60.35 \pm 56.40$ \\
TMJ Involvement (N\%) & $74.20 \pm 62.52$ \\
\end{tabular}

Table 2. Characteristics of the study groups

\begin{tabular}{cccc}
\hline & With TMJ involvement & Without TMJ involvement & P value \\
\hline Male/Female (N\%) & $4(6.6 \%) / 31(51.6 \%)$ & $7(11.6 \%) / 18(30 \%)$ & 0.48 \\
Age (years) & $34.67 \pm 13.43$ & $45.53 \pm 15.40$ & 0.39 \\
RF: Positive/Negative (N\%) & $33(55 \%) / 2(3.3 \%)$ & $15(25 \%) / 10(16.6 \%)$ & 0.02 \\
CRP (mg/L) & $22.6 \pm 9.1$ & $17.2 \pm 11.1$ & 0.01 \\
ESR (mm/h) & $32.2 \pm 11.4$ & $30.4 \pm 12.1$ & 0.10 \\
\hline
\end{tabular}




\begin{tabular}{|c|c|c|c|}
\hline & With TMJ involvement & Without TMJ involvement & Pvalue \\
\hline Disease duration (months) & $62.20 \pm 46.40$ & $58.82 \pm 42.60$ & 0.47 \\
\hline Treatment duration (months) & $76.30 \pm 60.40$ & $72.26 \pm 58.24$ & 0.29 \\
\hline DAS28 & $4.03 \pm 1.09$ & $3.48 \pm 0.95$ & 0.62 \\
\hline
\end{tabular}

There was no significant difference between the groups in terms of age, gender, disease duration, treatment duration, or DAS28. Only RF and CRP levels were correlated with TMJ involvement. Among 35 patients with TMJ involvement, the most common symptom was TMJ pain, followed by morning stiffness, articular noises, and muscle pain or spasm. As shown in Table 3, limitation of jaw movement was the least common symptom.

Table 3. Frequency of clinical evidence of TMJ involvement in RA patients

\begin{tabular}{ccc}
\hline Signs and symptoms & No. & \% \\
\hline TMJ pain (during mastication or opening/closing the mouth) & 23 & 65.71 \\
Morning stiffness & 21 & 60.00 \\
Articular noises (crepitus and clicking sounds) & 18 & 51.42 \\
Muscle pain or spasm & 15 & 42.85 \\
Limitation of jaw movement & 2 & 5.71 \\
\hline
\end{tabular}

\section{Discussion}

RA acts as a systemic etiological factor with major effects on the development of temporomandibular disorders. Because its clinical manifestations are often silent, TMJ involvement in patients with RA has been ignored [11]. In this regard, a previous study reported that three-quarters of RA patients had complaints of pain in the orofacial region, including arthralgia, myalgia, or both [12]. The prevalence of TMJ involvement in RA differs widely in the literature, possibly because of different examination types, selection of patients, different diagnostic techniques, or involvement criteria $[2,3]$.

TMJ complaints may be overshadowed by RA symptoms elsewhere in the body [4]. Various papers have highlighted the increased prevalence of TMJ symptoms and signs in patients with inflammatory rheumatic disorders such as RA. Therefore, this cross-sectional study evaluated the prevalence of TMJ involvement in RA patients who referred to the outpatient clinic of Loghman-Hakim Hospital. According to the literature [13], different imaging modalities, such as magnetic resonance imaging (MRI), computed tomography (CT), and cone-beam computed tomography (CBCT) have been used to assess TMJ. In the current study, a questionnaire and physical examination were used to assess TMJ involvement in order to avoid imposing a financial burden on patients.

In the present study, 35 of the 60 patients had some clinical evidence of TMJ involvement. In agreement with previous research $[3,5]$, the present findings showed that TMJ pain was the most frequent symptom. The present study also investigated laboratory indicators of RA (CRP, ESR, and RF), which might be related to the involvement of TMJ. A significant association was found between TMJ involvement, RF, and CRP, which is consistent with previous reports [10]. Conversely, some researchers [14] have found associations between TMJ involvement and ESR, which is in contrast with the current findings.

According to previous studies, TMJ involvement follows the same destructive path as in other joints and is directly correlated with the severity and duration of RA; therefore, the duration of RA is regarded as an aggravating factor for the involvement of TMJ $[15,16]$. This finding was not confirmed in the present study, and there was no significant difference between the two groups. Also, age, gender, and duration of treatment were not correlated with TMJ involvement, which is inconsistent with previous reports [10].

The current study did not investigate the radiographic imaging of patients, which can be considered as a limitation. 


\section{Conclusion}

Based on the present findings, CRP levels were high in RA patients with TMJ involvement, and TMJ pain was observed in almost half of the patients. There was a significant association between TMJ involvement and RF. Therefore, clinicians should incorporate TMJ examination in their clinical evaluations. Additionally, case-control and longitudinal studies on larger sample sizes can help in un- derstanding the involvement of TMJ and its evolution in patients with RA.

\section{Acknowledgments}

The authors are grateful to the staff at Vali-Asr Hospital and all the patients who assisted with this study.

\section{Conflict of Interest}

The authors declare no conflicts of interest. 


\section{References}

1. Jameson JL. Harrison's principles of internal medicine McGraw-Hill Education; 2018.

2. Silman AJ, Pearson JE. Epidemiology and genetics of rheumatoid arthritis. Arthritis Res 2002; 4(S3):S265-72. doi: $10.1186 / \operatorname{ar} 578$.

3. Savtekin G, Şehirli A. Rheumatoid arthritis in temporo-mandibular joint: A review. Niger J Clin Pract 2018, 21(10):1243-46. doi: 10.4103/njcp.njcp_117_18.

4. Aliko A, Ciancaglini R, Alushi A, Tafaj A, Ruci D. Temporomandibular joint involvement in rheumatoid arthritis, systemic lupus erythematosus and systemic sclerosis. Int $\boldsymbol{J}$ oral Maxillofac Surg 2011; 40(7):704-09. doi: 10.1016/j. ijom.2011.02.026.

5. Sodhi A, Naik S, Pai A, Anuradha A. Rheumatoid arthritis affecting temporomandibular joint. Contemp Clin Dent 2015; 6(1):124-27. doi: 10.4103/0976-237X.149308.

6. Sidebottom A, Salha R. Management of the temporomandibular joint in rheumatoid disorders. $\boldsymbol{B r} \boldsymbol{J}$ Oral Maxillofac Surg 2013; 51(3):191-98. doi: 10.1016/j. bjoms.2012.04.271.

7. Moen K, Bertelsen LT, Hellem S, Jonsson R, Brun JG. Salivary gland and temporomandibular joint involvement in rheumatoid arthritis: relation to disease activity. Oral Dis 2005; 11(1):27-34. doi: 10.1111/j.16010825.2004.01054.x.

8. Aceves-Avila F, Chávez-López M, Chavira-González J, Ramos-Remus C. Temporomandibular joint dysfunction in various rheumatic diseases. Reumatismo 2013; 65(3):12630. doi: 10.4081/reumatismo.2013.126.

9. Dworkin SF, LeResche L. Research diagnostic criteria for temporomandibular disorders: review, criteria, examinations and specifications, critique. J Craniomandib Disord $1992 ; 6(4): 301-55$
10. Celiker R, Gökçe-Kutsal Y, Eryilmaz M. Temporomandibular joint involvement in rheumatoid arthritis: Relationship with disease activity. Scand J Rheumatol 1995; 24(1):22-25. doi: 10.3109/03009749509095149.

11. Cordeiro PC, Guimaraes JP, de Souza VA, Dias IM, Silva JN, Devito KL. et al. Temporomandibular joint involvement in rheumatoid arthritis patients: association between clinical and tomographic data. Acta Odontol Latinoam 2016; 29(3):219-24

12. Crincoli V, Anelli MG, Quercia E, Piancino MG, Di Comite M. Temporomandibular Disorders and oral features in early rheumatoid arthritis patients: an observational study. Int J Med Sci 2019; 16(2):253-63. doi: 10.7150/ ijms. 28361 .

13. Franks AS. Temporomandibular joint in adult rheumatoid arthritis. A comparative evaluation of 100 cases. Ann Rheum Dis 1969; 28(2):139-45. doi: 10.1136/ ard.28.2.139.

14. Ozcan I, Ozcan K, Keskin D, Bahar S, Boyacigil S, Dere H. Temporomandibular joint involvement in rheumatoid arthritis: correlation of clinical, laboratory and magnetic resonance imaging findings. $\boldsymbol{B} \boldsymbol{E N T} 2008$; 4(1):19-24.

15. Symmons DP, Barrett EM, Bankhead CR, Scott DG, Silman AJ. The incidence of rheumatoid arthritis in the United Kingdom: results from the Norfolk Arthritis Register. Br J Rheumatol 1994; 33(8):735-39. doi: 10.1093/rheumatology/33.8.735.

16. Cunha CO, Pinto LMS, Mendonça LMd, Saldanha ADD, Conti ACdCF, Conti PCR. Bilateral asymptomatic fibrous-ankylosis of the temporomandibular joint associated with rheumatoid arthritis: a case report. Braz Dent J 2012; 23(6):779-82. doi: 10.1590/s0103-64402012000600025. 